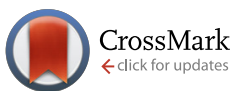

Cite this: Lab Chip, 2015, 15, 1545

Received 24th October 2014, Accepted 26th January 2015

DOI: $10.1039 / c 4 l c 01269 k$

www.rsc.org/loc

\title{
A microfluidic timer for timed valving and pumping in centrifugal microfluidics $\dagger$
}

\author{
F. Schwemmer, ${ }^{\text {*a }}$ S. Zehnle, ${ }^{\mathrm{b}}$ D. Mark, ${ }^{\mathrm{b}}$ F. von Stetten, ${ }^{\mathrm{ab}}$ R. Zengerle ${ }^{\mathrm{abc}}$ \\ and N. Paust ${ }^{a b}$
}

\begin{abstract}
Accurate timing of microfluidic operations is essential for the automation of complex laboratory workflows, in particular for the supply of sample and reagents. Here we present a new unit operation for timed valving and pumping in centrifugal microfluidics. It is based on temporary storage of pneumatic energy and time delayed sudden release of said energy. The timer is loaded at a relatively higher spinning frequency. The countdown is started by reducing to a relatively lower release frequency, at which the timer is released after a pre-defined delay time. We demonstrate timing for 1 ) the sequential release of 4 liquids at times of $2.7 \mathrm{~s} \pm 0.2 \mathrm{~s}, 14.0 \mathrm{~s} \pm 0.5 \mathrm{~s}, 43.4 \mathrm{~s} \pm 1.0 \mathrm{~s}$ and $133.8 \mathrm{~s} \pm 2.3 \mathrm{~s}, 2$ ) timed valving of typical assay reagents (contact angles $36-78^{\circ}$, viscosities $0.9-5.6 \mathrm{mPa}$ s) and 3 ) on demand valving of liquids from 4 inlet chambers in any user defined sequence controlled by the spinning protocol. The microfluidic timer is compatible to all wetting properties and viscosities of common assay reagents and does neither require assistive equipment, nor coatings. It can be monolithically integrated into a microfluidic test carrier and is compatible to scalable fabrication technologies such as thermoforming or injection molding.
\end{abstract}

\section{Introduction}

Centrifugal microfluidics is a powerful tool for automation of bio-chemical assays ${ }^{1-4}$ with significant advantages when compared to other microfluidic automation concepts: artificial gravity by centrifugation inherently removes bubbles that might interfere with suitable assay performance and the centrifugal propulsion allows for the automation of complex assay protocols without any interfaces to external valves and pumps. One important challenge, however, is the automation of the precise timing of fluidic operations, such as reagent supply or valving after an incubation period. The timed addition of reagents for example is required for diverse assay types such as immunoassays, DNA/RNA extraction and amplification, DNA sequencing etc.

In centrifugal microfluidics, timed valving independent of the rotational frequency protocol has been demonstrated

\footnotetext{
${ }^{a}$ Laboratory for MEMS Applications, IMTEK - Department of Microsystems Engineering, University of Freiburg; Georges-Koehler-Allee 103, 79110 Freiburg, Germany. E-mail: frank.schwemmer@imtek.de

${ }^{b}$ HSG-IMIT - Institut für Mikro- und Informationstechnik, Georges-Koehler-Allee 103, 79110 Freiburg, Germany

${ }^{c}$ BIOSS - Centre for Biological Signaling Studies, University of Freiburg, 79110 Freiburg, Germany

$\dagger$ Electronic supplementary information (ESI) available: Details on the network simulation and theory of priming of the pneumatic siphons. See DOI: 10.1039/ c4lc01269k
}

using valves actuated by external lasers or infrared light sources, ${ }^{5-8}$ external pressure sources ${ }^{9,10}$ or external mechanical actuation. ${ }^{11}$ While active valves allow for elegant fluidic automation, the trade-off is a more complex processing device and in most cases additional fabrication steps for production of the disposable cartridge.

Timing without employing external means, also referred to as passive timing, can be realized by employing capillary forces. Such passive timing is commonly used in capillary flow based microfluidics, by designing fluidic resistances in combination with surface tension-based passive pumping or geometric valves. ${ }^{12,13}$

In centrifugal microfluidics, passive valves are typically triggered at increasingly high rotational frequencies. For this purpose, geometric valves, ${ }^{14}$ hydrophobic patches ${ }^{15}$ and centrifugo-pneumatic valves ${ }^{16-18}$ are used. Another passive solution combines a geometric valve triggered at high rotational frequencies with a siphon valve primed by capillary forces at low rotational frequencies. ${ }^{19,20}$ Furthermore, the recently introduced miniature-stick-packs can be used for sequential release of pre-stored liquids at pre-defined rotational frequencies. ${ }^{21}$ However, all of these passive valves for timed release strongly depend on fabrication tolerances. The geometric valves and hydrophobic patches additionally depend on capillary forces. The accurate control of such dependencies is challenging and leads to considerable variations in rotational burst frequencies, as for example discussed 
by van Oordt et $a .^{21}$ for reagent release. For a sequential supply of reagents according to the assay protocol, the trigger frequencies have to be sufficiently high to prevent pre-mature release, for example during transport or storage of the cartridge; the frequencies must increase with the release sequence and must not overlap with respect to its variations. As a consequence, sequential release of more than three liquids is a challenging task, in particular, if robust operation within cartridges that are compatible to cost efficient mass fabrication technologies is required.

Recently, a new type of passive timing independent of the rotational frequency based on dissolvable films was introduced. ${ }^{17}$ The dissolvable films are used to either block a fluidic path or an air vent. Upon contact with the liquid, the film starts to dissolve and after a certain time period, the path opens. By clever combination of dissolvable films with liquid and air routing, Kinahan et al. demonstrated automation of up to 10 sequential valving steps. ${ }^{22}$ However, the fabrication of such cartridges requires an extra fabrication step for introducing the dissolvable films and the dissolvable film is dissolved within the assay reagents. While the authors could show that for a PCR-based assay and an immunoassay, the dissolved film does not impact results, ${ }^{17}$ the use of such valves changes the composition of the assay, which can lead to issues for established assays.

This paper introduces the microfluidic timer as a new fully passive fluidic unit operation for precise temporal control of valving and pumping of typical assay reagents and samples. The timer can easily be combined with any unit operation, where actuation is achieved by compression and decompression of entrapped air volumes. Examples of such pneumatic operations are mixing by reciprocating flow, ${ }^{23,24}$ inward pumping $^{25,26}$ or centrifugo-pneumatic cascading. ${ }^{27}$ The principle of the microfluidic timer is based on centrifugal pressures, pneumatic pressures and viscous dissipation, only. Therefore, the microfluidic timer is not restricted to any specific materials and can be fabricated monolithically with established fabrication technologies such as injection molding or thermoforming. Monolithic fabrication in this context means that the timer can be implemented within the same substrate, without additional fabrication steps, simply by structuring the timer features with the same technology as the rest of the microfluidic features.

We provide a theoretical model that can be used to accurately predict the delay time by network simulations in order to adapt release times to the specific assay needs. Additionally, the model is simplified to enable a more rough analytical prediction of delay times which in our opinion is sufficient for most implementations. The model is validated by comparing simulated timed valving to experimental data from different liquids that cover a wide range of typical assay reagents.

Experimentally we discuss a sequential release, and a release on demand mode. For the latter, within a given design of a microfluidic disk hosting four different liquids, routing of the four input liquids on demand in any user defined sequence is presented.

\section{General fluidic principle and design rules}

\section{Functional principle of the microfluidic timer}

In theory, the timer can be applied for all microfluidic platforms ${ }^{4}$ that provide pressure control. This paper will discuss the application of the microfluidic timer in centrifugal microfluidics, in which pressure control is achieved by centrifugation. The principle of the microfluidic timer is based on temporary storage of pneumatic energy and time delayed sudden release of said energy. The main components of the timer are two pneumatic chambers which are connected to each other by a capillary with a defined fluidic resistance (Fig. 1). To load the pneumatic energy, a loading pressure is applied to pump liquid into the pneumatic chambers compressing the entrapped air. At a critical filling, the first pneumatic chamber is overfilled; the timing channel is primed and liquid fills the second pneumatic chamber such that the timer is loaded (Fig. 1A). Subsequently, the loading pressure is turned off and liquid is slowly pushed out of pneumatic chamber 2 by the pressurized entrapped air. The flow rate of liquid is thus limited due to the viscous pressure drop $p_{\text {visc }}$ along the timing channel (Fig. 1B). After a predefined time period, all liquid has left pneumatic chamber 2 and the resistance of the timing channel suddenly changes due to the viscosity change from liquid to air ( 50 fold for water/air). At this moment, the timer is released. The flow rate suddenly increases and abruptly releases the stored pneumatic energy. The sudden energy release can be used as a trigger for valving, pumping or other operations (Fig. 1C) as demonstrated in detail in the experimental section.

\section{Theoretical description of the timer}

This section describes how the delay time and the stored pneumatic energy of the microfluidic timer can be calculated analytically. The stored pneumatic energy $E_{\text {pneu }}$ which is released abruptly upon timer release can be calculated by:

$$
E_{\text {pneu }}=\int_{0}^{V \text { pneu } 1} p_{\text {pneu }} \mathrm{d} V
$$

with $V_{\text {pneu1 }}$ as the volume of pneumatic chamber 1 , and $p_{\text {pneu }}$ as the overpressure inside the pneumatic chambers.

The delay time is the time it takes for all of the liquid to flow out of pneumatic chamber 2. The start of the delay time is defined when the pressure applied for loading and setting the timer is turned off or significantly reduced.

The delay time ends at the timer release, when air replaces the liquid in the timing channel, abruptly changing the fluidic resistance and rapidly releasing the remaining stored pneumatic energy. For the case that the loading pressure is turned off completely, the delay time can be derived from a pressure balance between the pneumatic pressure difference 


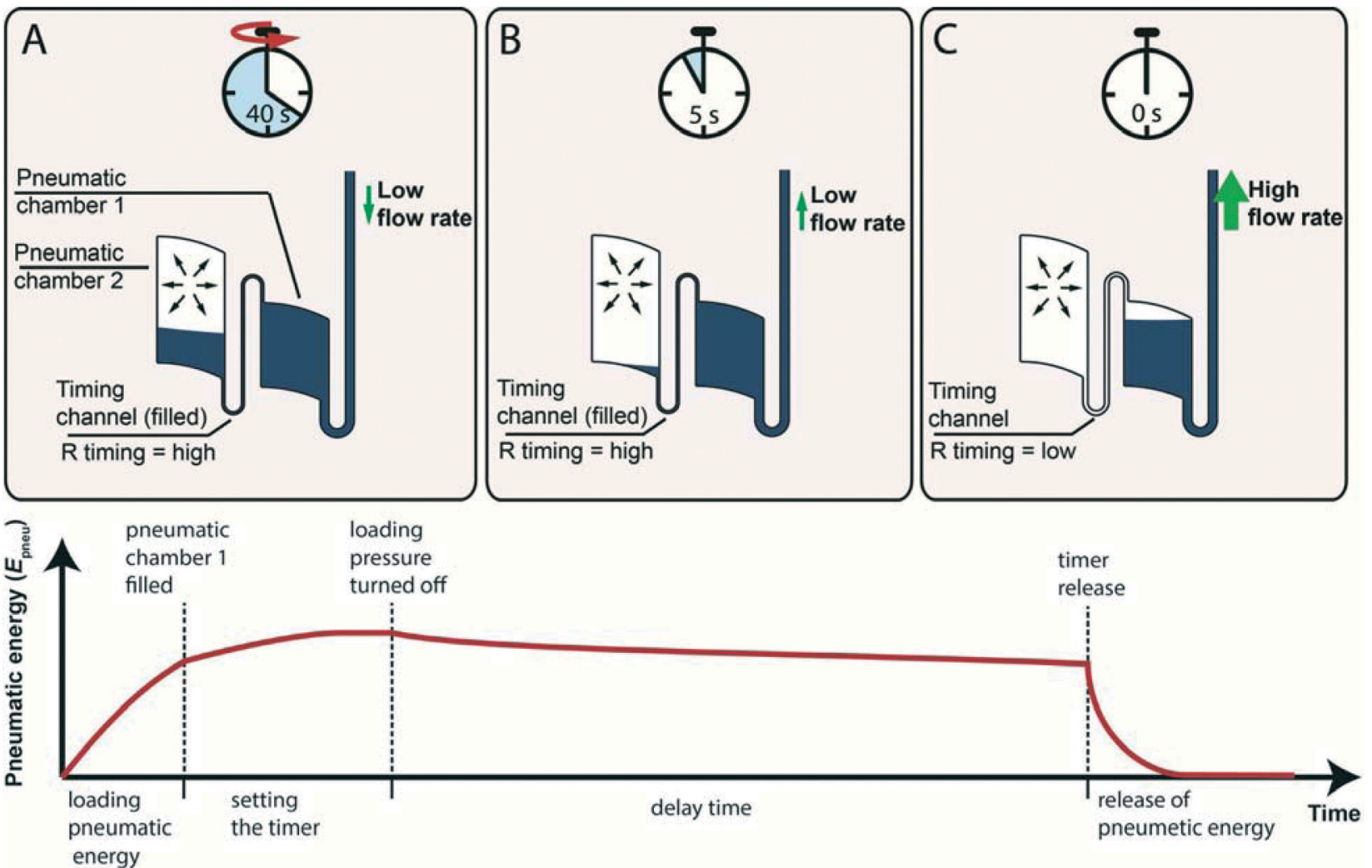

Fig. 1 Functional principle of the microfluidic timer (upper) and stored pneumatic energy in the microfluidic timer during different steps of its operation (lower). A: Timer loading by applying a loading pressure. Air is compressed within the pneumatic chamber and the pneumatic energy is loaded. After pneumatic chamber 1 is completely filled, liquid overflows into pneumatic chamber $2 a$. The liquid volume transferred into pneumatic chamber $2 \mathrm{a}$ sets the timer, which defines the length of delay time. B: Delay time. The applied loading pressure is turned off and the pneumatic pressure pushes liquid out of pneumatic chamber 2a. The high viscous dissipation in the timing channel limits the flow rate. During this period, the pneumatic energy is released very slowly. C: Timer release. After the timing channel has been emptied of liquid, the flow rate temporarily increases by the viscosity ratio of liquid and gas and the pneumatic energy is abruptly released. This energy release can be employed to transport liquid e.g. for valving or pumping. One practical example for this is the priming of a siphon in centrifugal microfluidics, as further detailed in the next section (see Fig. 2).

$p_{\text {pneu }}$ of the enclosed air and the viscous pressure drop $p_{\text {visc }}$ along the timing channel:

$$
\begin{gathered}
p_{\text {visc }}\left(\frac{\mathrm{d} V_{\text {comp }}}{\mathrm{d} t}\right)=p_{\text {pneu }}\left(V_{\text {comp }}\right) \\
R \frac{\mathrm{d} V_{\text {comp }}}{\mathrm{d} t}=\frac{p_{\text {atm }} V_{0}}{V_{0}-V_{\text {comp }}}-p_{\text {atm }}
\end{gathered}
$$

This differential equation can be solved for the delay time:

$$
\Delta t_{\text {delay }}=\frac{R V_{0}}{p_{\text {atm }}} \ln \left(\frac{V_{\text {pneul }}}{V_{\text {pneul }}+V_{\text {loaded }}}\right)+\frac{R}{p_{\text {atm }}} V_{\text {loaded }}
$$

where $R$ represents the fluidic resistance of the timing channel, $p_{\text {atm }}$ is the atmospheric pressure, $V_{0}$ is the total volume of pneumatic chambers $1 \& 2, V_{\text {pneu1 }}$ is the volume of pneumatic chamber 1 and $V_{\text {loaded }}$ is the volume of liquid in pneumatic chamber 2 at the start of the delay.

For design of the microfluidic timer, eqn (4) can be used to calculate the delay time (see Tables $1 \& 2$ ). The parameters used for designing the delay time are the fluidic resistance of the timing channel, the volumes of pneumatic chambers 1 $\& 2$, and the liquid volume in pneumatic chamber 2 at the start of the delay. For a liquid of viscosity $\eta$ the fluidic resistance $R$ of a rectangular channel of length $l$, height $h$, and width $w$ can be calculated for $w>h$ by:

$$
R=\frac{\alpha \eta l}{w h^{3}}
$$

$$
\alpha=12\left[1-\frac{192 h}{\pi^{5} w} \tanh \left(\frac{\pi w}{2 h}\right)\right]^{-1}
$$

where $\alpha$ is a dimensionless parameter, which solely depends on the aspect ratio of the microchannel. ${ }^{28}$

From eqn (4) \& (5) it can be seen that the delay time is linearly related to the fluidic resistance $R$, which in turn scales linearly with the length $l$ of the delay channel. The delay time also scales linearly with respect to the volumes of pneumatic chambers $1 \& 2$ and the liquid volume in pneumatic chamber 2 at the start of the delay, as long as all three 
volumes are scaled by the same factor. Obviously, an increase in volume can thus be compensated for by decreasing the length of the delay channel for design of a specific delay time.

\section{Application of the timer in centrifugal microfluidics}

Since timed sequential addition of reagents is required for many assays, we choose the timed release of liquids as an application example for the microfluidic timer. For this, we implement the timer for temporal controlled valving of a pneumatic siphon valve as depicted in Fig. 2A. An inlet structure for reagent supply branches into the timer and a siphon channel, which in turn is connected to a collection chamber. The microfluidic timer is loaded at high centrifugation $(f=$ $85 \mathrm{~Hz}$, Fig. 2B-C). The resulting high hydrostatic pressure pushes the liquid from the inlet chamber into the pneumatic chambers 1 \& 2. Upon deceleration to the timer release frequency, the hydrostatic pressure decreases, causing liquid to slowly flow out of chamber $2 \mathrm{a}$ and back into the inlet chamber (Fig. 2D). After a defined delay time, the liquid in the timing channel is replaced with air and the timer is released, causing a high flow rate out of pneumatic chamber 1. The flow out of pneumatic chamber 1 is split into the siphon channel and the inlet channel. If a critical volume $V_{\text {crit }}$ is pumped into the siphon channel, the siphon primes (Fig. 2E). Since the crest of the siphon is located radially inwards of the inlet channel, priming must be performed against centrifugal forces based on a dynamic principle. The underlying effect is that the sudden increase in the flow rate upon timer release causes an increase in viscous dissipation in the inlet channel. This leads to radially inward flow in the siphon channel as employed for pneumatic inward pumping, ${ }^{25}$ which allows for priming the siphon even against centrifugal or capillary forces. A detailed description of siphon priming, based on a pressure balance according to Kirchhoff's law, is provided in the ESI. $\dagger$

After priming, the siphon transfers the sample to the collection chamber (Fig. 2F). In order to transfer the complete sample to the collection chamber, it is beneficial to maintain a moderate rotational frequency at timer release ( $\sim 15 \mathrm{~Hz}$ ) (Fig. 2F). The moderate rotational frequency maintains a centrifugal pressure difference from the inlet chamber to the collection chamber and thus allows for transferring the complete liquid volume to the collection chamber. In such configurations, we define the start of the delay time as the time when the moderate rotational frequency is reached. The maintained centrifugal pressure counteracts the pneumatic pressure and reduces the pressure drop across the timing channel. As a consequence, the liquid outflow through the timing channel decreases and the delay time increases. To account for the increase in delay time, the following equation should be solved:

$$
p_{\text {cent }}\left(V_{\text {comp }}\right)+p_{\text {visc }}\left(\frac{\mathrm{d} V_{\text {comp }}}{\mathrm{d} t}\right)=p_{\text {pneu }}\left(V_{\text {comp }}\right)
$$

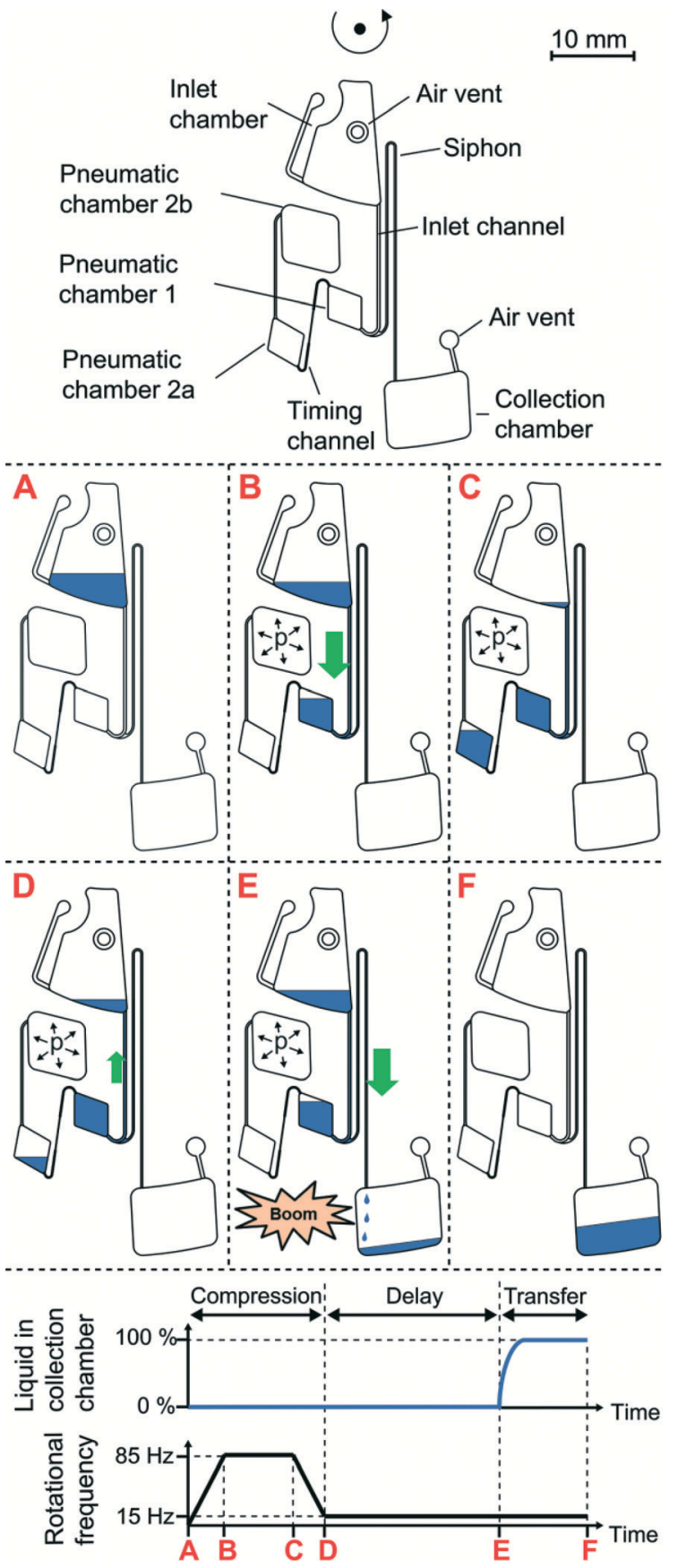

Fig. 2 Implementation of the microfluidic timer into a centrifugal microfluidic disk. Green arrows indicate the liquid flow rate. A: Liquid is pipetted into the inlet chamber. B: At high rotational frequency, the liquid is transferred from the inlet chamber to the pneumatic chambers. C: The first pneumatic chamber is overfilled, the timing channel is primed and the timer is loaded until the gas overpressure equilibrates the centrifugal pressure. D: Upon lowering centrifugation, the fluidic resistance of the timer channel limits the maximal flow and delays the expansion of the entrapped gas volume. E: Pneumatic chamber $2 a$ has run empty and the timer is released. The fluidic resistance of the delay channel reduces abruptly as the viscosity within the channel drops from liquid to the one of air. This results in a sudden increase in flow rate. The flow is split towards the siphon channel and the inlet channel. At low centrifugation a critical volume of liquid flows into the siphon channel. The siphon primes and transfers the sample in the collection chamber. F: The sample liquid is transferred to the collection chamber. 
This equation includes the centrifugal pressure term $p_{\text {cent }}$ ( $\left.V_{\text {comp }}\right)$ that depends on the filling heights of the different chambers and thus on the integrated flow rate and geometry of the timer. It can only be solved numerically. For that purpose we use a network simulation based approach which was described previously, ${ }^{25}$ and which is further detailed in the ESI. $\uparrow$ With this network based simulation, we can predict liquid flow and fill levels throughout the fluidic processing. If the centrifugal pressure $p_{\text {cent }}$ is much smaller than the pneumatic pressure $p_{\text {pneu }}$, the analytical estimation from eqn (4) can be used as a good approximation, as described further in the results section.

\section{Materials \& methods}

All fluidic structures were designed using SolidWorks (Concord, MA, USA) and fabricated by the Lab-on-a-Chip Design- \& Foundry-Service of HSG-IMIT (www.loac-hsg-imit. de/en/design-foundry-service). Microstructures were milled in a $4 \mathrm{~mm}$ PMMA substrate (Plexiglas, Evonik, Germany) using a precision micro-mill (KERN Microtechnik GmbH). The disks were then cleaned by $\mathrm{CO}_{2}$ blasting, rinsed with DI water and isopropyl alcohol and subsequently dried using nitrogen gas. The PMMA disks were sealed via lamination of polyolefin adhesive tape (\# 900320, HJ Bioanalytik, Germany). Experiments were performed using a prototype LabDiskPlayer (Qiagen Lake Constance $\mathrm{GmbH}$ ) that was modified to a stroboscopic setup (BioFluidix $\mathrm{GmbH}$ ). Where not otherwise noted, all given viscosities were measured using a rheometer (Anton Paar Physica MCR 101). Experiments were performed at room temperature $\left((24 \pm 1){ }^{\circ} \mathrm{C}\right)$.

Due to the high pressures in the pneumatic chambers, the sealing foil buckles outwards. This deformation of the sealing foil increases the volume of the pressure chamber and decreases the pneumatic pressure. We calculated the actual pneumatic pressure from the rotational frequency and height of the liquid column at maximal rotation. All given volumes for the pressure chambers are measured. All calculations and simulations are based on these measured volumes for the pneumatic chambers.

\section{Experimental results}

\section{Sequential release}

Four different variations of the microfluidic timer were implemented with varying dimensions (Table 1). The volume of pneumatic chamber 1 and pneumatic chamber $2 \mathrm{a}$ is $46.7 \mu \mathrm{l}$, each. The timer inlets were filled with $100 \mu$ l of DI water, each. The timers were then loaded at $85 \mathrm{~Hz}$ centrifugation. After pneumatic chambers 1 \& 2 were filled and a steady state was reached, the centrifugation frequency was reduced to $15 \mathrm{~Hz}$ with a deceleration rate of $15 \mathrm{~Hz} \mathrm{~s}^{-1}$. From the stroboscopic image data, the transferred sample volume to the four collection chambers was measured over time. All experiments were performed in triplicate. The measured delay times during the experimental series are listed in Table 1 . The sequential release of the four timers can be seen in Fig. 3. The network simulation based on eqn (7) accurately predicted the measured delay times. The analytical estimation from eqn (4) underestimates the delay time. This is expected, since the analytical model ignores the counteracting centrifugal force, which acts against the pneumatic pressure. If longer delay times than the reported $\sim 2$ min are required, an adaption of the frequency protocol can be applied to release liquids on demand, as further detailed in the release on demand section.

\section{Typical assay reagents}

The delay time for the microfluidic timer depends linearly on the viscosity of the timed liquid. Thus, for the same timer structure, the liquid with the lowest viscosity would be released first. However, in general, liquids need to be released in any order, independent of their viscosities. To demonstrate viscosity-independent sequential release, we show timed valving of typical biological reagents in opposite order of their viscosities. We used timers 1, 3, and 4 with geometries as listed in Table 1.

The timers were filled such that the liquid with the highest viscosity $\left(50 \%\right.$ glycerol $\mathrm{w} / \mathrm{w}, \theta=(78 \pm 6)^{\circ}, \eta=(5.62 \pm$ $0.02) \mathrm{mPa} \mathrm{s}$, at $24{ }^{\circ} \mathrm{C}$ ) was released first, then the liquid with medium viscosity was valved $(50 \% \mathrm{w} / \mathrm{w}$ ethanol in DI water, $\theta=(36 \pm 8)^{\circ}, \eta=(2.56 \pm 0.02) \mathrm{mPa} \mathrm{s}$, at $\left.24^{\circ} \mathrm{C}\right)$ and finally, the liquid with the lowest viscosity was released (blood plasma $\eta \sim 1.68 \mathrm{mPa}$ ). As shown in Fig. 3, all liquids could be sequentially released in opposite order of their viscosities, with the delay times calculated by the network simulation (see ESI $\dagger$ ).

To determine the viscosity of blood plasma, we used the microfluidic timer as a rheometer by iterative adaption of viscosity in the network simulation to fit the measured delay time. The resulting viscosity of $\sim 1.68 \mathrm{mPa}$ s falls well within

Table 1 Implementations of the microfluidic timer in the microfluidic disk. The length of the timer channel was $13.8 \mathrm{~mm}$. The volume of pneumatic chamber $1\left(V_{\text {pneu1 }}\right)$ and the volume in pneumatic chamber $2 a$ at the start of the delay period $\left(V_{\text {loaded }}\right)$ was $46.7 \mu l$ each. All measured and calculated delay times are for DI water at $24^{\circ} \mathrm{C}$

\begin{tabular}{lllll}
\hline & Timer 1 & Timer 2 & Timer 3 & Timer 4 \\
\hline Cross section of the timing channel in $\mu \mathrm{m} \times \mu \mathrm{m}$ & $153 \times 125$ & $106 \times 67$ & $80 \times 41$ & $71 \times 24$ \\
Total volume of pneumatic chambers $V_{0}$ in $\mu \mathrm{l}$ & 357 & 278 & 1.98 & 38.7 \\
Delay time estimated by eqn (4) in s & 1.9 & 13.2 & 43.6 & 123.6 \\
Delay time from network simulation in s & 2.2 & $14.0 \pm 0.5$ & $43.4 \pm 1.0$ & 133.3 \\
Measured delay time in s & $2.7 \pm 0.2$ & $133.8 \pm 2.3$
\end{tabular}



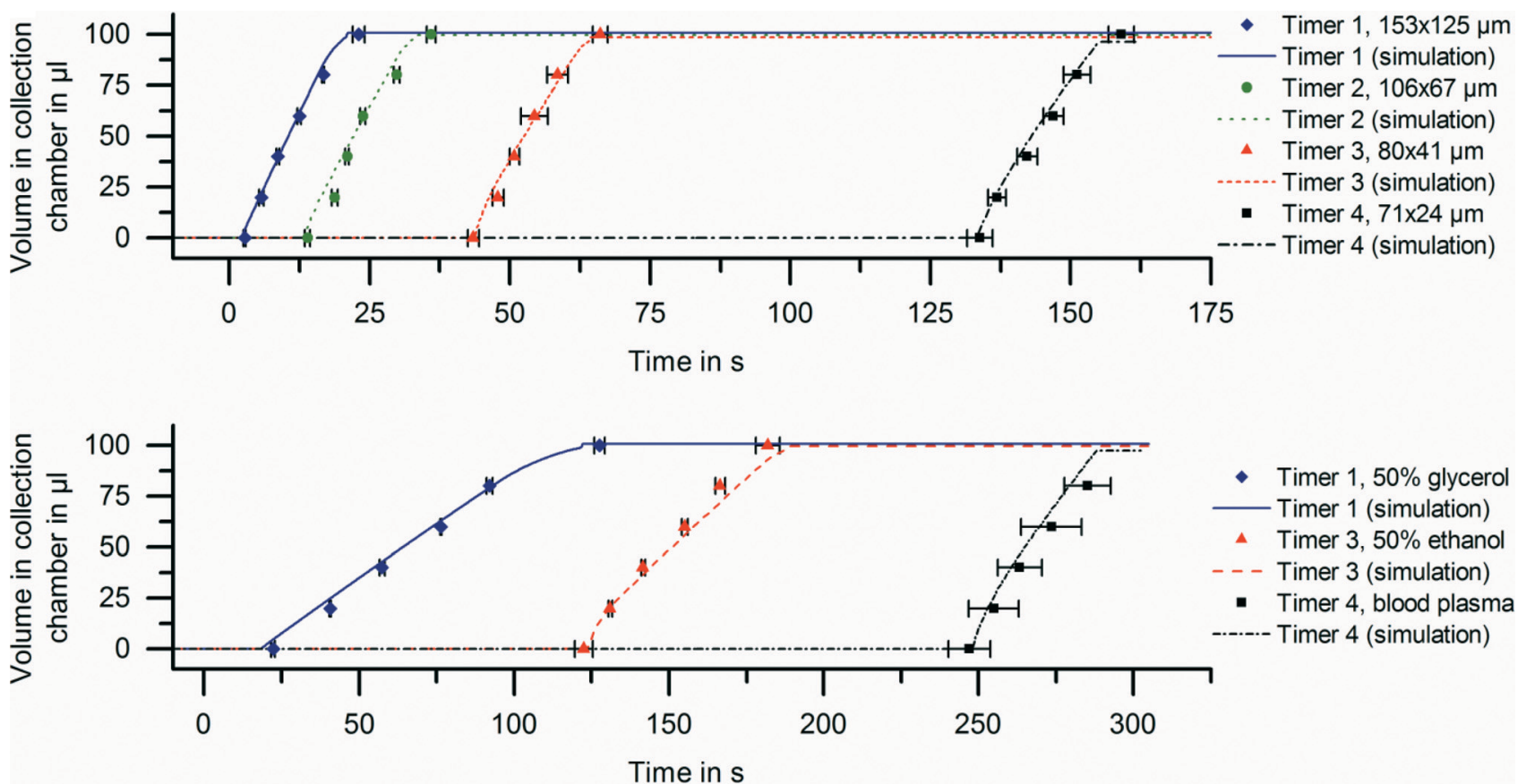

Fig. 3 Sequential release with the microfluidic timer. (Upper) Release of DI water at RT for the four different timer structures (Table 1). The timers were engineered to release at sequential points in time for DI water. (Lower) Timing of typical assay reagents and sequential release in opposite order of their viscosity demonstrated employing timers 1, 3 and 4. The delay time and the time to transfer the sample to the collection chamber depend on the viscosity of the sample. All experiments were performed in triplicate. The network simulations are described in detail in the ESI $\dagger$ (see Fig. S1). Error bars represent one standard deviation.

the reference range for a healthy adult, which is reported to be $1.52-1.76 \mathrm{mPa}$ s at $24^{\circ} \mathrm{C} .^{29}$

\section{Release on demand}

For the previous timing events, a minimal frequency protocol has been applied: timer loading at a constant high rotational frequency and subsequent release at constant low rotation. Using a more complex frequency protocol, the functionality of the timer can be extended to a release on demand feature. That means that each of the timing structures can be addressed and released individually and independently from each other. This way valving can be performed in any user defined sequence allowing for $N$ ! valving combinations. This for example results in 24 combinations with $N=4$ timing structures. The underlying working principle can be explained as follows.

As discussed previously, during timer release, the viscosity within the timing channel suddenly changes from liquid to air, resulting in a temporarily high flow and consequently, a higher viscous pressure drop in the inlet channel. This pressure drop leads to pumping of liquid radially inwards through the siphon channel, against the centrifugal force as employed previously by Zehnle et al. for pneumatic inward pumping. ${ }^{25}$ Since the centrifugal force can be adjusted by the frequency protocol upon timer release, it is possible to determine on demand whether the siphon does or does not prime. If a comparatively high rotational frequency is applied at the moment of timer release, siphon priming can be inhibited because the centrifugal force limits the amount of liquid pumped into the siphon. Under those circumstances the critical volume for priming the siphon is not reached and no liquid is transferred to the collection chamber. Applying a comparatively low rotational frequency at the moment of timer release, siphon priming is promoted as more liquid is pumped into the siphon channel. Under those circumstances the siphon primes and liquid is transferred to the collection chamber (see ESI $\dagger$ ). Consequently, with an appropriate frequency protocol, it is possible to selectively valve exactly one out of multiple timing structures on demand without influencing all the others.

We demonstrate the valve on demand mode with a microfluidic disk containing four microfluidic timers that are

Table 2 Implementation of the microfluidic timer for release on demand. The cross section of the timer channel was $52 \times 43 \mu \mathrm{m}$. The total volume of the pneumatic chambers $V_{0}$ was $211 \mu$. The volume of pneumatic chamber $1\left(V_{\text {pneu1 }}\right)$ and the volume in pneumatic chamber $2 a$ at the start of the delay period $\left(V_{\text {loaded }}\right)$ was $46.7 \mu$ l each. All experiments were replicated 10 times. All measured and calculated delay times are for DI water at $24^{\circ} \mathrm{C}$

\begin{tabular}{lllll}
\hline & Timer 1 & Timer 2 & Timer 3 & Timer 4 \\
\hline $\begin{array}{l}\text { Length of timing channel } \\
\text { in mm }\end{array}$ & 1 & 2 & 3 & 4 \\
$\begin{array}{l}\text { Delay time estimated by } \\
\text { eqn (4) in s }\end{array}$ & 5.2 & 10.3 & 15.5 & 20.6
\end{tabular}



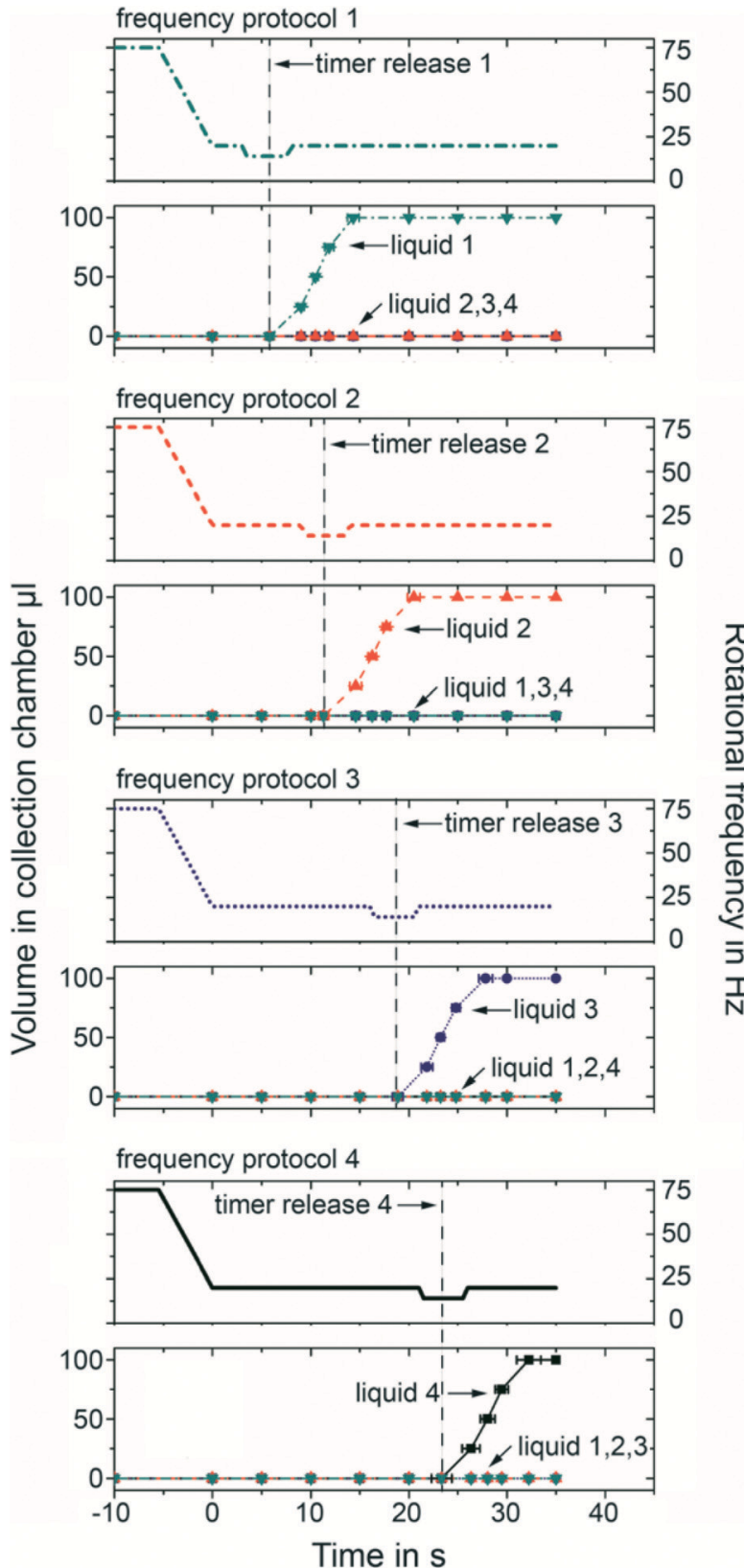

Fig. 4 Release on demand with four microfluidic timers with increasing delay times: $t_{\text {delay } 1}=5.8 \mathrm{~s} \pm 0.4 \mathrm{~s}$, $t_{\text {delay } 2}=11.3 \mathrm{~s} \pm 0.5 \mathrm{~s}$, $t_{\text {delay } 3}=18.8 \mathrm{~s} \pm 0.5 \mathrm{~s}, t_{\text {delay } 4}=23.4 \mathrm{~s} \pm 1.0 \mathrm{~s}$. After loading the timers at $75 \mathrm{~Hz}$, centrifugation is reduced to $20 \mathrm{~Hz}$. In each of the experiments, one of the siphon valves could be selectively primed by reducing the frequency further to $14 \mathrm{~Hz}$ shortly before timer release and increasing back to $20 \mathrm{~Hz}$ shortly after. As depicted, all other siphon valves did not prime and the liquid is transferred back to the inlet chamber, instead. This allows us to sequentially release the four microfluidic timers in any user defined sequence. Each curve corresponds to ten experiments. The data were collected for fixed transferred volumes between 0 and $100 \mu \mathrm{l}$ in $25 \mu \mathrm{l}$ steps. Error bars correspond to the time variations when a discrete volume $(25 \mu \mathrm{l})$ has been transferred. The largest standard deviation was $\pm 1.2 \mathrm{~s}$ measured for frequency protocol 4 in $100 \mu \mathrm{l}$ volume in the collection chamber. All other error bars are smaller and thus hardly visible in the graphs. released at four different delay times. The layout was designed using eqn (4) making use of the linear relationship between the delay time and the lengths of the timing channels (Table 2).

The microfluidic timers are designed such that after loading the timer, the microfluidic timers can release at all rotational frequencies below $28 \mathrm{~Hz}$. Moreover, if the timer release happens at a rotational frequency of $14 \mathrm{~Hz}$ or below, the siphons prime. In contrast, if the timer release happens at a rotational frequency of $16 \mathrm{~Hz}$ and above, the siphons do not prime. That way timer release and valving are independently controllable.

All four timers were filled with $100 \mu \mathrm{l}$ of DI water and the timers were loaded at $75 \mathrm{~Hz}$ rotational frequency. The delay time starts after a reduction of rotational frequency to $20 \mathrm{~Hz}$ by $15 \mathrm{~Hz} \mathrm{~s}^{-1}$. In order to prime one siphon valve, the rotational frequency was reduced to $14 \mathrm{~Hz}$ just before the predicted timer release of the corresponding timer. After priming of the siphon, the rotational frequency was increased back to $20 \mathrm{~Hz}$. This "frequency dip" allowed us to selectively valve the siphon of one of the microfluidic timers, while all siphons of all other timers remained not primed. Fig. 4 shows the results for selective priming of siphons of each of the microfluidic timers. All of the experiments were repeated ten times. Selective transfer of the sample from one of the timers could be shown successfully in all 40 experiments. Since the initial loaded state could be restored by rotating at a high rotational frequency of $75 \mathrm{~Hz}$ (timer reset), this allowed us to transfer the samples from the microfluidic timers to the collection chambers in any user defined sequence by simply concatenating frequency protocols 1-4 according to user needs. In the future this may allow us to use one fluidic disk design for multiple assay protocols, by simply adjusting the frequency protocol to a different application.

It is worth noting that if one timer is released multiple times in sequence, liquid plugs can form in the siphon channel. Due to contact angle hysteresis this can increase the capillary pressure to a level, for which the siphon channel does no longer prime, even below $14 \mathrm{~Hz}$ rotation upon timer release. Such liquid plugs can be easily removed from channels by including an intermediate step at high rotational frequency above the Rayleigh Taylor instability. ${ }^{18}$ In our case, the critical rotational frequency to remove plugs from the $150 \mu \mathrm{m}$ diameter siphon channel was $\sim 80 \mathrm{~Hz}$ and an intermediate increase in rotational frequency to $90 \mathrm{~Hz}$ was included. The temporary high centrifugation ensured that no liquid plugs remained in the siphon channel.

\section{Conclusion}

We introduced a microfluidic timer that can be used for timed release of liquid reagents with different properties such as viscosities and surface tensions. It can easily be integrated with existing unit operations, and does not 
require surface treatments, assistive equipment or additional fabrication steps.

By timing the release of pneumatically stored energy, the microfluidic timer circumvents a major limitation of many state of the art centrifugo-pneumatic unit operations that require fast changes in the rotational frequency. So far, fast frequency changes had to be provided by the processing device. ${ }^{25}$ In the future, employing a microfluidic timer, fast acceleration or deceleration rates are not required anymore to enable a quick release of pneumatic energy. Thus, it allows for centrifugo-pneumatic operations, such as pneumatic inward pumping, to be used in a much wider range of processing devices, e.g. standard laboratory centrifuges as used for example for the LabTube technology. ${ }^{30}$

Furthermore, the microfluidic timer enables to trigger valving after a specific delay time and this way enables to reduce or increase the spinning frequencies while the countdown of the timer is running. This creates an additional degree of freedom compared to state of the art valves, e.g., combining siphon based valving with the microfluidic timer allows for applying spinning frequencies during the countdown of the timer which are not accessible for state of the art capillary siphons or geometric- or centrifugopneumatic valves because those frequencies would initiate unintended valving.

Finally, the demonstrated capabilities of the timer in release on demand mode aim towards a universal cartridge design. With release on demand, a generic cartridge can automate timed valving for different assay protocols, simply by programming the protocol of the rotational frequency.

\section{Outlook}

We expect the microfluidic timer to find broad applications within centrifugal microfluidic automation of sequential on demand release of reagents. Moreover, the timer could also be used as a trigger after stopping the disk enabling the release of the stored pneumatic energy with a defined flow rate with the disk at rest. This is useful to perform measurements under fluid flow with a stationary detector, for example for particle counting or impedance spectrometry on a disk.

Furthermore, when using at increased temperatures, the microfluidic timer can be combined with a vapor-diffusion barrier to reduce air pressure and risk of delamination. ${ }^{31}$

Finally, the microfluidic timer can be generalized to other microfluidic areas besides centrifugal microfluidics, since it allows precise flow sequencing for multiple liquids with pressure sources that are comparatively simple, e.g. fingeractuated devices. ${ }^{32}$

\section{Acknowledgements}

We gratefully acknowledge financial support from the Federal Ministry of Education and Research (BMBF) in the project SAXS-CD (Verbundforschungsprojekt, project number 05K10VFB).

\section{Notes and references}

1 J. Ducrée, S. Haeberle, S. Lutz, S. Pausch, F. von Stetten and R. Zengerle, J. Micromech. Microeng., 2007, 17, S103.

2 R. Gorkin, J. Park, J. Siegrist, M. Amasia, B. S. Lee, J.-M. Park, J. Kim, H. Kim, M. Madou and Y.-K. Cho, Lab Chip, 2010, 10, 1758-1773.

3 M. Madou, J. Zoval, G. Jia, H. Kido, J. Kim and N. Kim, Annu. Rev. Biomed. Eng., 2006, 8, 601-628.

4 D. Mark, S. Haeberle, G. Roth, F. von Stetten and R. Zengerle, Chem. Soc. Rev., 2010, 39, 1153-1182.

5 K. Abi-Samra, R. Hanson, M. Madou and R. A. Gorkin III, Lab Chip, 2011, 11, 723-726.

6 W. Al-Faqheri, F. Ibrahim, T. H. G. Thio, J. Moebius, K. Joseph, H. Arof and M. Madou, PLoS One, 2013, 8, e58523.

7 J. L. Garcia-Cordero, D. Kurzbuch, F. Benito-Lopez, D. Diamond, L. P. Lee and A. J. Ricco, Lab Chip, 2010, 10, 2680-2687.

8 B. S. Lee, Y. U. Lee, H.-S. Kim, T.-H. Kim, J. Park, J.-G. Lee, J. Kim, H. Kim, W. G. Lee and Y.-K. Cho, Lab Chip, 2011, 11, 70-78.

9 M. C. R. Kong and E. D. Salin, Anal. Chem., 2010, 82, 8039-8041.

10 M. C. R. Kong and E. D. Salin, Anal. Chem., 2011, 83, 1148-1151.

11 T. Kawai, N. Naruishi, H. Nagai, Y. Tanaka, Y. Hagihara and Y. Yoshida, Anal. Chem., 2013, 85, 6587-6592.

12 M. W. Toepke, V. V. Abhyankar and D. J. Beebe, Lab Chip, 2007, 7, 1449-1453.

13 M. Zimmermann, P. Hunziker and E. Delamarche, Microfluid. Nanofluid., 2008, 5, 395-402.

14 R. D. Johnson, I. H. A. Badr, G. Barrett, S. Lai, Y. Lu, M. J. Madou and L. G. Bachas, Anal. Chem., 2001, 73, 3940-3946.

15 Y. Ouyang, S. Wang, J. Li, P. S. Riehl, M. Begley and J. P. Landers, Lab Chip, 2013, 13, 1762-1771.

16 C. E. Nwankire, D.-S. S. Chan, J. Gaughran, R. Burger, R. Gorkin and J. Ducrée, Sensors, 2013, 13, 11336-11349.

17 R. Gorkin III, C. E. Nwankire, J. Gaughran, X. Zhang, G. G. Donohoe, M. Rook, R. O'Kennedy and J. Ducrée, Lab Chip, 2012, 12, 2894-2902.

18 D. Mark, T. Metz, S. Haeberle, S. Lutz, J. Ducrée, R. Zengerle and F. von Stetten, Lab Chip, 2009, 9, 3599-3603.

19 C. E. Nwankire, G. G. Donohoe, X. Zhang, J. Siegrist, M. Somers, D. Kurzbuch, R. Monaghan, M. Kitsara, R. Burger and S. Hearty, Anal. Chim. Acta, 2013, 781, 54-62.

20 J. Siegrist, R. Gorkin, L. Clime, E. Roy, R. Peytavi, H. Kido, M. Bergeron, T. Veres and M. Madou, Microfluid. Nanofluid., 2010, 9, 55-63.

21 T. van Oordt, Y. Barb, J. Smetana, R. Zengerle and F. von Stetten, Lab Chip, 2013, 13, 2888-2892.

22 D. J. Kinahan, S. M. Kearney, N. Dimov, M. T. Glynn and J. Ducrée, Lab Chip, 2014, 14, 2249-2258.

23 Z. Noroozi, H. Kido, M. Micic, H. Pan, C. Bartolome, M. Princevac, J. Zoval and M. Madou, Rev. Sci. Instrum., 2009, 80, 075102 . 
24 M. M. Aeinehvand, F. Ibrahim, W. Al-Faqheri, T. H. G. Thio, A. Kazemzadeh and M. Madou, Lab Chip, 2014, 14, 988-997.

25 S. Zehnle, F. Schwemmer, G. Roth, F. von Stetten, R. Zengerle and N. Paust, Lab Chip, 2012, 12, 5142-5145.

26 K. Abi-Samra, L. Clime, L. Kong, R. Gorkin III, T.-H. Kim, Y.-K. Cho and M. Madou, Microfluid. Nanofluid., 2011, 11, 643-652.

27 N. Godino, R. Gorkin III, A. V. Linares, R. Burger and J. Ducrée, Lab Chip, 2013, 13, 685-694.

28 C. J. Morris and F. K. Forster, Exp. Fluids, 2004, 36, 928-937.
29 N. K. Shinton, CRC desk reference for hematology, CRC Press, Boca Raton, 1998.

30 A. Kloke, A. R. Fiebach, S. Zhang, L. Drechsel, S. Niekrawietz, M. M. Hoehl, R. Kneusel, K. Panthel, J. Steigert, F. von Stetten, R. Zengerle and N. Paust, Lab Chip, 2014, 14, 1527-1537.

31 G. Czilwik, I. Schwarz, M. Keller, S. Wadle, S. Zehnle, F. von Stetten, D. Mark, R. Zengerle and N. Paust, Lab Chip, 2014, DOI: 10.1039/C4LC01115E.

32 W. Li, T. Chen, Z. Chen, P. Fei, Z. Yu, Y. Pang and Y. Huang, Lab Chip, 2012, 12, 1587-1590. 